Objectives In this study we compared the clinical efficacy of cyclosporin A (Cyc-A) and interferon-alfa (INF-alfa) in our patients with severe posterior uveitis.

Methods All patients were classified and followed by means of fundus fluoressein angiography. At baseline, Cyc-A and INF-alfa were given in a dose of $5 \mathrm{mg} / \mathrm{kg} /$ day and 4,5 million $U$ three times a week, respectively. In case of an attack, a prednison treatment course was added those monotherapy regimen. Twenty-one patients were included in the study. Eight and eleven patients were started on Cyc-A and INF-alfa regimens, respectively. Here, the results at the 3- and 6-month duration were presented.

Results Two of 8 patients treated with Cyc-A were stopped to continue due to inefficacy and switched on INF-alpha therapy. In the same group one patient had gingival hyperplasia, which partially responded to dose tapering, however another patient with azotemia and hypertension had to be changed to other treatment group. Except one, all patients on INF-alpha regimen completed the study with adequate regression of inflammatory activity. In that patient depression unresponsive to drugs leaded to stop INF-alpha at the end of 6 months.

Conclusion Although it is difficult to make a clear conclusion about the comperative efficacy of INF-alpha and Cyc-A due to small population and short follow-up. But it seems that INF-alfa more tolerable than Cyc-A in the treatment of Behçet' Disease with severe ocular involvement.

\section{OP0042 COLOUR DUPLEX ULTRASONOGRAPHY IN THE DIAGNOSIS OF TEMPORAL ARTERITIS}

${ }^{1} \mathrm{G}$ Nesher, ${ }^{2} \mathrm{D}$ Shemesh, ${ }^{1} \mathrm{M}$ Mates, ${ }^{3} \mathrm{M}$ Sonnenblick, ${ }^{2} \mathrm{HB}$ Abramowitz. ${ }^{1}$ Internal Medicine; ${ }^{2}$ Vascular Surgery; ${ }^{3}$ Geriatrics, Shaare-Zedek Medical Center, Jerusalem, Israel

10.1136/annrheumdis-2001.230

Background The diagnosis of temporal arteritis (TA) usually requires a temporal artery biopsy. Recently it has been reported that a periluminal dark halo, detected by colour duplex ultrasonography (CDU) of the temporal arteries, is a characteristic sign of TA, and commonly appears in these patients.

Objectives To examine the predictive value of the dark halo sign in diagnosing TA.

Methods During a period of 2 years 69 patients suspected of having TA were examined by CDU of both temporal arteries, using the Acuson SEQUOIA 512 with a $7 \mathrm{MHz}$ linear array transducer. Temporal artery biopsy was performed in 32 of the patients. The diagnosis of TA was made if a patient had a positive biopsy, or met all of the 3 following criteria: 1. ACR classification criteria were fulfilled, 2. there was a prompt clinical response to $40-60 \mathrm{mg} /$ day of prednisone, 3. no other diagnosis related to the symptoms was made during a follow-up of 6 months.

Results Periluminal dark halo was observed in 24 of the 69 patients. TA was diagnosed in 12 of them, giving a positive predictive value (PPV) of only 50\%. No halo was detected in 45 cases. Only 2 of them had TA, giving a high negative predictive value (NPV) of $96 \%$. The sensitivity and specificity of the halo sign for diagnosing TA were $86 \%$ and $78 \%$, respectively.

Conclusion The PPV of the halo sign in CDU of the temporal arteries is unsatisfactory for diagnosing TA. However, The NPV is very high. Thus a negative halo sign can practically serve to rule out a diagnosis of TA and precludes the need for a biopsy in most instances.
OP0043 A STUDY OF OVERLAPING INFECTIOUS, AUTOIMMUNE AND HAEMATOLOGICAL ETIOLOGIES IN CRYOGLOBULINEMIA IN A TERTIARY UNIVERSITARY HOSPITAL

${ }^{1} \mathrm{M}$ Ramos-Casals, 'O Trejo, 'M Garcia-Carrasco, ${ }^{2} \mathrm{~J}$ Yague, ${ }^{1} \mathrm{G}$ De la Red, 'S Jimenez, ${ }^{1} \mathrm{Ml}$ Quenard, ${ }^{1} \mathrm{R}$ Cervera, ${ }^{1} \mathrm{~J}$ Font, ${ }^{1} \mathrm{M}$ Ingelmo. 'Systemic Autoimmune Diseases Unit; ${ }^{2}$ Department of Immunology, Hospital Clinic, Barcelona, Spain

10.1136/annrheumdis-2001.231

\section{Background}

Objectives To study the main etiological factors associated to cryoglobulinemia in a universitary hospital, to determine the percentage of patients with no evidence of associated disease (? essential? cryoglobulinemia) and to analyse the amount of overlaping between the different etiologies.

Methods We analysed 443 consecutive patients with cryoglobulinemia, who tested positive for circulating cryoglobulins in our Department of Immunology between 1991 and 1999. Of these 443 patients, 258 (58\%) were women and 185 (42\%) men (mean age 54 years, range 14 to 91 ).

Results Infectious diseases were detected in 331 (75\%) patients, autoimmune diseases in 94 (24\%), haematological disease in 33 (7\%) and essential cryoglobulinemia in 49 (11\%). Patients with autoimmune diseases showed a cryocrit higher than 5\% (26\%) more frequently compared with those with haematological processes (24\%), HCV infection (16\%) or essential cryoglobulinemia $(2 \%)(\mathrm{p}=0.003)$. HCV infection was found in $321(73 \%)$ patients, HBsAg in $15(3 \%)$ and HIV antibodies in $29(7 \%)$. Forty (9\%) patients had SJÖGREN'S syndrome (SS), 30 (7\%) systemic lupus erythematosus (SLE), 7 (2\%) periarteritis nodosa and $6(2 \%)$ progressive systemic sclerosis. Finally, $16(4 \%)$ patients had a NHL, 3 chronic lymphocytic leukaemia, 3 multiple myeloma and 2 Hodgking lymphoma. When the overlaping of more than one etiological factor was analysed, we found that most patients with HBsAg or HIV infection were also HCV positive. In addition, we observed a strong overlap between HCV and some autoimmune (50\% in SS, 67\% in PAN) or haematological $(50 \%$ of NHL) diseases.

Conclusion HCV infection was the main etiological factor identified, being present in $73 \%$ of patients with cryoglobulinemia. Of the systemic autoimmune diseases, SS and SLE were those frequently associated to cryoglobulinemia, and NHL was the most frequent haematological process found. Associated disease was not found in $11 \%$ of patients (essential cryoglobulinemia). Finally, we report a strong overlap between certain etiological factors (HCV, SS and NHL).

\section{OP0044 EPIDEMIOLOGY OF VASCULITIS IN THREE REGIONS OF EUROPE}

${ }^{1} \mathrm{RA}$ Watts, ${ }^{2} \mathrm{~W}$ Koldingsnes, ${ }^{2} \mathrm{H}$ Nossent, ${ }^{3} \mathrm{MA}$ Gonzalez-Gay, ${ }^{3} \mathrm{C}$ Garcia-Porrua, ${ }^{4} \mathrm{G}$ Bentham, ${ }^{5} \mathrm{SE}$ Lane, ${ }^{5} \mathrm{DG}$ Scott. ${ }^{1}$ Rheumatology, Ipswich Hospital, Ipswich; ${ }^{2}$ Rheumatology, University of Tromso, Tromso, Norway; ${ }^{3}$ Rheumatology, Hospital XeralCalde, Lugo, Spain; ${ }^{4}$ Environmental Sciences, University of Tromso; ${ }^{5}$ Rheumatology, Norfolk and Norwich Hospital, Norwich, UK

10.1136/annrheumdis-2001.232

Background The epidemiology of systemic vasculitis (SV) has been poorly documented. Development of accepted criteria and definitions by the ACR and Chapel Hill Consensus Conference (CHCC) permits comparison of data from different areas. We 